\title{
Analysis of CSMA/CA Systems under Carrier Sensing Error: Throughput, Delay and Sensitivity
}

\author{
Jo Woon Chong, Youngchul Sung, and Dan Keun Sung \\ School of EECS, KAIST \\ 373-1 Guseong-dong, Yuseong-gu, Daejeon 305-701, KOREA \\ E-mail: jw9607@gmail.com, \{ysung, dksung\}@ee.kaist.ac.kr
}

\begin{abstract}
In this paper, the performance of the carrier sense multiple access/ collision avoidance (CSMA/CA) protocol under the presence of carrier sensing error is analyzed. Based on our previous work [1], we extend the results to $n$-user case $(n>2)$, and analyze the sensitivity of the throughput with respect to the key physical-layer parameter, the sensing threshold, via a cross-layer approach. The result provide guidelines about how to operate the CSMA/CA considering imperfect sensing at physical layer. It is shown that the throughput sensitivity highly depends on the ratio of the contention window size $W$ to the frame length $L$, and the throughput is sensitive to the design of the sensing threshold when $W / L$ is either small or large.
\end{abstract}

\section{INTRODUCTION}

The carrier sense multiple access/ collision avoidance (CSMA /CA) is a well-established random access scheme [2], and has been used widely. In CSMA/CA, each user senses the channel to determine the availability of channel, and accesses the channel depending on the sensing outcome. Recently, the protocol gains renewed attention since it is adopted in many wireless systems such as WLAN and ZigBee [3],[4], and several authors conducted the accurate analysis of CSMA/CA performance to design more efficient system parameters for the protocol [5],[6],[7],[8]. In these analyses, however, it is assumed that no carrier sensing errors exist, and the physical layer property is not properly captured. Moreover, recently Krishnamurthy et al. measured the aggregated throughput of the CSMA/CA system by varying the carrier sensing threshold, which determines the sensing error probabilities, in an experiment, and found that the aggregated throughput drastically changed according to the carrier sensing threshold value [9]. In this paper, we extend our previous work [1] of the crosslayer approach to the analysis of the CSMA/CA to $n$-user case, and investigate the impact of the carrier sensing error at the physical later to the throughput and delay. Further, we consider commonly used carrier sensing method, the energy detector, and investigate the sensitivity of the throughput with respect to the sensing threshold. It is shown that the sensitivity of the throughput mainly depends on the ratio of the contention window size to the frame size, and the results provide guideline for the cross-layer design for CSMA/CA.

\section{SySTEM MODEL}

In this section, we briefly introduce the system model that is also presented in [1].

\section{A. Operation of CSMA/CA stations under the presence of sensing error}

We consider a network consisting of $n$ transmitter-receiver pairs operating with the CSMA/CA protocol. To facilitate the analysis, we assume that the system is slotted and synchronized among stations. We also assume that the transmitters have only one backoff stage and the contention window $(\mathrm{CW})$ size of the backoff stage is $W$, and the CSMA/CA stations are in saturated mode, i.e., they always have frames to transmit. In the slotted CSMA/CA each transmitter senses the channel at the beginning of each slot. If the level of the sensor's input signal is larger than a pre-determined threshold, the CSMA/CA station determines that the channel is busy. Otherwise, it determines that the channel is idle. However, the sensing is not perfect and there are sensing errors because of channel impairment such as noise or distortion during the transmission. There are two types of sensing error: false alarm and miss detection errors. A false alarm error is defined as an error which occurs when the sensor determines that the channel is busy when the channel is actually idle. On the other hand, a miss detection error is defined as an error which occurs when the sensor determines that the channel is idle provided that the channel is actually busy. The false alarm probability and the miss detection probability, denoted by $p_{f}$ and $p_{m}$, respectively, are typically dependent on the input signal power, the noise power, the sensing threshold value, and the sensor type.

Fig. 1 illustrates the operation of the slotted CSMA/CA. Before a station initiates the transmission of a frame, it senses the channel to determine the availability of the channel. If the channel is sensed to be idle for a backoff unit slot $\sigma$, the value of the backoff counter decreases as in Fig. 1. Otherwise, the station continues sensing the channel until the channel is sensed to be idle for an interval of $\sigma$. In Fig. 1, Station $B$ experiences both miss detection and false alarm errors. During Period 1, Station $B$ decreases the value of its backoff counter from 5 to 4 since Station $B$ miss-detects the transmission of station $A$. During Period 2, on the other hand, Station $B$ freezes its backoff counter since Station $B$ has a false alarm error.

The operation of a CSMA/CA station is modeled using a Markov chain with states being the values of the backoff counter. Fig. 2 shows the proposed Markov chain model for the operation of each CSMA/CA station capturing the false 


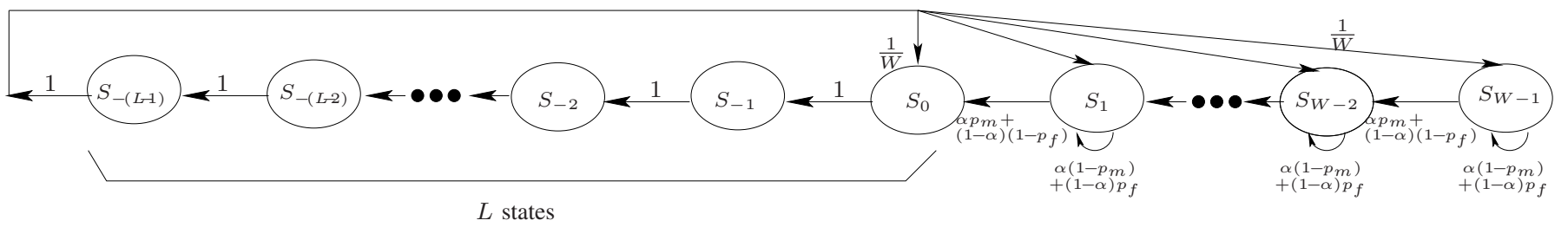

Fig. 2. Markov chain model for a CSMA/CA node with sensing errors $\left(0 \leq p_{f}, p_{m} \leq 1\right)[1]$



Fig. 1. Operation of CSMA/CA Basic Access Protocol

alarm and miss detection errors at the sensing stage [1]. Here, the state transition occurs every slot. The chain is divided into the right and left sides around State $S_{0}$. State $S_{i}$ in the right part represents that the CSMA/CA station has a backoff counter value of $i$ without transmitting a frame. State $S_{i}$ in the left part corresponds to the case that the CSMA/CA station is transmitting $(-i+1)^{t h}$ slot of a frame with a backoff counter value of 0 . In the states of the right part of the chain, the given station decreases its current backoff counter value by one 1) when the given station determines that the channel is idle (with probability $1-p_{f}$ ) when it is actually idle (with probability $1-\alpha$, where $\alpha$ is the channel activity factor), or 2 ) when the given station declares that the channel is idle (with probability $p_{m}$ ) when it is actually busy (with probability $\alpha$ ). Otherwise, the given station freezes its current backoff counter. In the states of the left part of the chain, the CSMA/CA station is in the process of transmitting a frame with length of $L$ slots. Whether the transmission of each slot of the frame is successful or not, the state of the CSMA/CA station moves to the left. If there is no collision during the transmission over the $L$ slot period, we consider that the frame is successfully transmitted. If at least one of the $L$ slots of the frame is collided, on the other hand, we consider that the frame is collided.

The state transition probabilities for the Markov chain in Figure 2 are given by

$$
\begin{array}{lc}
P\left\{S_{i-1} \mid S_{i}\right\}=\alpha p_{m}+(1-\alpha)\left(1-p_{f}\right), & i \in[1, W-1], \\
P\left\{S_{i} \mid S_{i}\right\}=\alpha\left(1-p_{m}\right)+(1-\alpha) p_{f}, & i \in[1, W-1], \\
P\left\{S_{(i-1)} \mid S_{i}\right\}=1, & i \in[-(L-2), 0], \\
P\left\{S_{i} \mid S_{-(L-1)}\right\}=1 / W, & i \in[0, W-1],
\end{array}
$$

where $W$ denotes the contention window size. We denote the stationary probability of state $S_{i}$ by $b_{i}$. From the above transition probabilities, we obtain the relationship between stationary probabilities given by

$$
\begin{array}{ll}
b_{i}=b_{0}, & i \in[-(L-1),-1], \\
b_{i}=\frac{b_{0}}{\alpha p_{m}+(1-\alpha)\left(1-p_{f}\right)} \frac{W-i}{W}, & i \in[1, W-1],
\end{array}
$$

and the normalization condition for the stationary distribution is given by

$$
1=b_{0}\left\{L+\frac{1}{\alpha p_{m}+(1-\alpha)\left(1-p_{f}\right)} \frac{W-1}{2}\right\} .
$$

From the above equations, we obtain the stationary probability $b_{0}$ for State $S_{0}$ as a function of $p_{f}, p_{m}, W, L$, and $\alpha$, given by

$$
b_{0}\left(p_{f}, p_{m}, W, L, \alpha\right)=\frac{2\left\{\alpha p_{m}+(1-\alpha)\left(1-p_{f}\right)\right\}}{2 L\left\{\alpha p_{m}+(1-\alpha)\left(1-p_{f}\right)\right\}+(W-1)} .
$$

Stationary probabilities for other states are obtained using (1) based on $b_{0}$ in Eqn. (3).

\section{B. Collision probability $p_{c}$ and channel activity factor $\alpha$}

Fig. 3 shows the operation of a CSMA/CA network with two pairs of stations under the presence of carrier sensing errors for illustration. Stations $A$ and $B$ are transmitting data to stations $C$ and $D$, respectively. The transmission of data from one transmitter to its corresponding receiver $(A \rightarrow C$ or $B \rightarrow C$ ) is successful if no other transmission occurs during the transmission (see periods 1 and 3 ). If two transmitters $A$ and $B$ start to transmit their data frames simultaneously, collision occurs (period 2). This event happens even in the perfect sensing CSMA/CA network when the backoff counters values of more than two stations reach 0 simultaneously as well as in the imperfect sensing CSMA/CA network. However, the transmission attempt by transmitter $A$ to $C$ in the middle of the transmission by transmitter $B$ to $D$ (period 4) or the opposite case (period 5) occurs only in the network with imperfect sensing. Hence, these additional collisions need to be considered in modeling the operation of the CSMA/CA model under the presence of carrier sensing error.

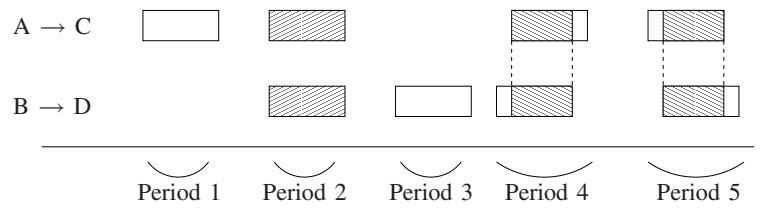

Fig. 3. Data transmission flow time diagram of two pairs of stations in a CSMA/CA network with sensing error 
Generally, in $n$-user case the transmission of one station is collided, in the middle, if some other transmitter is in State $S_{1}$ and miss-detect the channel. (For example, see Period 5 with Station A as the station that has on-going transmission in Fig. 3.) We denote by $p_{c_{l}}$ the probability that a transmitted frame of one station is collided from the $l^{\text {th }}$ slot due to the transmission by some other station in the network, and assume that $p_{c_{l}}$ is the same irrespective of the value of $l,(2 \leq l \leq L)$ except the case of $l=1$. We denote it by $p_{c}\left(=p_{c_{2}}=p_{c_{3}}=\cdots=p_{c_{L}}\right)$, and $p_{c}$ is given by

$p_{c}=1-\left(1-\frac{b_{1}}{\sum_{i=1}^{W-1} b_{i}} \times p_{m}\right)^{n-1}=1-\left(1-\frac{2 p_{m}}{W}\right)^{n-1}$,

where $n$ is the total number of transmitters in the network. Note that $\frac{b_{1}}{\sum_{i=1}^{W-1} b_{i}} \times p_{m}$ is the probability that another transmitter (say, Station B) among $n-1$ transmitters other than the station having the on-going transmission starts transmission from the back-off stage (conditioned that there is already a transmission going on.) The condition that one transmitter is transmitting without collision up to $(l-1)$ th slot of the frame, implies that all other stations (including Station B) are in the back-off stage up to this stage. $b_{1} \times p_{m}$ explains the probability of the further event that the back-off counter value of Station $\mathrm{B}$ is one and Station B mis-detects the channel.

Since $\alpha$ is the probability that the channel is busy from the viewpoint of a particular station, $\alpha$ is the probability that at least one of other stations is in one of the states in left part of Markov chain, and is given by

$$
\alpha=1-\left(\sum_{i=1}^{W-1} b_{i}\right)^{n-1},
$$

where $\left(\sum_{i=1}^{W-1} b_{i}\right)^{n-1}$ is the probability that all $n-1$ other transmitters are in the back-off stage, i.e., the right side of the chain in Figure 2.

\section{AnAlysis of CSMA/CA Under the PRESENCE of CARRIER SENSING ERRORS}

\section{A. Normalized throughput $S$ and mean access delay $D$}

Our approach to calculate the total system throughput is based on a slot-by-slot approach. That is, we calculate for each slot the probability that the slot is used for successful transmission, collision or idle period. We define $P_{b o}$ as the probability that a station has a positive backoff counter value ( $b_{i}$ where $1 \leq i \leq W-1$ ). We also define $\tau$ as the probability that a station initiates transmission conditioned that the station has a positive backoff counter value and the channel is not occupied. Then, $P_{b o}$ and $\tau$ are given by

$$
\begin{gathered}
P_{b o}=\sum_{i=1}^{W-1} b_{i}, \\
\tau=\frac{b_{1}}{\sum_{i=1}^{W-1} b_{i}}\left(1-p_{f}\right),
\end{gathered}
$$

where $p_{f}$ is the false alarm probability. Under the condition, a station initiates transmission if and only if the station is in State $S_{1}$ and does not make an false alarm error, which explains Eqn. (7).

The successful transmission, collision, and idle probabilities for the given station, denoted by $P_{S}, P_{C}$, and $P_{I}$, respectively, are obtained as functions of $P_{b o}, \tau, p_{c}$, and $L$ using the stationary distribution, and are given by

$$
\begin{aligned}
& P_{I}=P_{b o}^{n}(1-\tau)^{n}, \\
& P_{S}=n \times P_{b o}^{n} \tau(1-\tau)^{n-1} \times\left(1-p_{c}\right)^{L-1} L, \\
& P_{C}=1-P_{S}-P_{I} .
\end{aligned}
$$

Here, $P_{b o}{ }^{n}$ represents the probability that the channel is idle at the beginning of the slot, and Eqn. (8) is obvious. Eqn. (9) is explained as follows. $P_{b o}^{n} \tau(1-\tau)^{n-1}$ is the probability that the channel is idle at the beginning of the slot and one and only one user initiates the transmission of a frame. However, the first slot of the frame would not be regarded as a successfully transmitted slot unless the following $L-1$ slots are also successfully transmitted. This explains the term $\left(1-p_{c}\right)^{L-1}$, and the term $L$ is the normalization factor. Once the event of successful transmission of a frame occurs, total $L$ slots are consumed. Factor $n$ represents that the successful transmission of a frame can be done by any of the $n$ transmitters in the network. Thus, the normalized throughput $S$ of the CSMA/CA is given by

$$
S=\frac{\sigma P_{S}}{\sigma\left(P_{I}+P_{S}+P_{C}\right)},
$$

where $\sigma$ is the slot length. From Equations (10) and (11) $S$ is given by

$$
S=n P_{b o}{ }^{n} \tau(1-\tau)^{n-1}\left(1-p_{c}\right)^{L-1} \times L,
$$

Substituting (4), (6), and (7) into (12) yields

$$
\begin{aligned}
S= & n\left[\frac{1}{\left\{1-\left(1-L b_{0}\right)^{n-1}\right\} p_{m}+\left(1-L b_{0}^{n-1}\right)\left(1-p_{f}\right)} \frac{W-1}{2}\right]^{n}(13) \\
& {\left[\frac{2\left(1-p_{f}\right)}{W}\right]\left[1-\frac{2\left(1-p_{f}\right)}{W}\right]^{n-1}\left[\left(1-\frac{2 p_{m}}{W}\right)^{n-1}\right]^{L-1} \times L . }
\end{aligned}
$$

where $b_{0}$ is obtained from the solution of (3) and is a function of $p_{m}, p_{f}, W$ and $L$. Note that $S$ is a function of various physical/MAC layer parameters such as $p_{m}, p_{f}, W, L$, and $n$.

The delay can be easily obtained by Little's law. Let $D$ be the average access delay, defined as the time elapsing between the instant of time that the frame is put into service - i.e., it becomes head-of-line (HOL) - and the instant of time that the frame terminates a successful deliver. $D$ is given, using Little's law, by [6].

$$
D=\frac{E[n]}{S / E[P]}
$$

where $P$ is the payload length (i.e., the frame size in our case) the numerator $E[n]$ represents the average number of competing stations which will successfully deliver their HOL frame, and the denominator $S / E[P]$ represents the frame delivery rate (i.e., the throughput measured in frames/seconds). 


\section{Sensitivity ANalysis: An Example of ENERgy} DETECTOR

\section{A. Receiver operating characteristics (ROC) of an energy} detector

As the carrier sensing method for a CSMA/CA node, either a matched filter or an energy detector can be used. The matched filter detector requires the knowledge of the signal signature whereas the energy detector does not. In this section, we consider the commonly used energy detector and analyze the sensitivity of the throughput and delay with respect to the sensing threshold.

Assuming that both signal and noise are modeled as white Gaussian process, the problem is modeled statistically by the following hypotheses for the observation.

$$
\begin{cases}H_{0} & : \quad Y \sim \mathcal{N}\left(0, \sigma_{0}^{2}\right), \\ H_{1} & : \quad Y \sim \mathcal{N}\left(0, \sigma_{0}^{2}+\sigma_{1}^{2}\right),\end{cases}
$$

where $Y$ is an observation sample, and $\sigma_{0}^{2}$ and $\sigma_{1}^{2}$ are noise power and the primary signal power, respectively. ${ }^{*}$ Under the Neyman-Pearson criterion, the false alarm probability $p_{f}$ and the miss detection probability $p_{m}$ for the energy detector is given by [10]:

$$
\begin{aligned}
& p_{f}=\epsilon(\eta)=1-\Gamma\left(\frac{1}{2}, \frac{\eta}{2 \sigma_{0}^{2}}\right), \\
& p_{m}=\delta(\eta)=\Gamma\left(\frac{1}{2}, \frac{\eta}{2\left(\sigma_{0}^{2}+\sigma_{1}^{2}\right)}\right),
\end{aligned}
$$

where $\Gamma(m, a)=\frac{1}{\Gamma(m)} \int_{0}^{a} t^{m-1} e^{-t} d t$ is the incomplete gamma function.

The operating characteristics of a detector is typically described by the ROC curve that shows the miss detection probability $p_{m}$ as a function of the false alarm probability $p_{f}$. Fig. 4 is the ROC of an energy detector for several sets of the input signal power $\sigma_{1}^{2}$, the noise power $\sigma_{0}^{2}$ and the sensing threshold $\eta$. The increase of the sensing threshold for given signal and noise power moves the operating point toward upper direction along one of the curves in the figure. Note that, at the very high SNR regime in Fig. 4, either of $p_{m}$ or $p_{f}$ can maintain low values even if the sensing threshold threshold changes much. This is not the case in the low SNR.

\section{B. Sensitivity of throughput $\frac{d S}{d \eta}$}

The sensitivity is a measure of the degree of variation of its performance from nominal, due to changes in the elements constituting the system. We define the sensitivity of the normalized throughput $S$ with respect to the change in the sensing threshold $\eta$ as $\frac{d S}{d \eta}$. Since $S$ is a function of $p_{m}$ and $p_{f}$, the sensitivity of the throughput $\frac{d S}{d \eta}$ can be expressed as

$$
\left|\frac{d}{d \eta} S\right|=\left|\frac{\partial S}{\partial p_{f}} \frac{d p_{f}}{d \eta}+\frac{\partial S}{\partial p_{m}} \frac{d p_{m}}{d \eta}\right| .
$$

Note that the overall sensitivity consists of the two terms: the detector characteristics $\left(\frac{d p_{f}}{d \eta}\right.$ are $\left.\frac{d p_{m}}{d \eta}\right)$ and throughput

\footnotetext{
${ }^{*} \mathcal{N}\left(0, \sigma^{2}\right)$ denotes the zero-mean Gaussian distribution with variance $\sigma^{2}$.
}

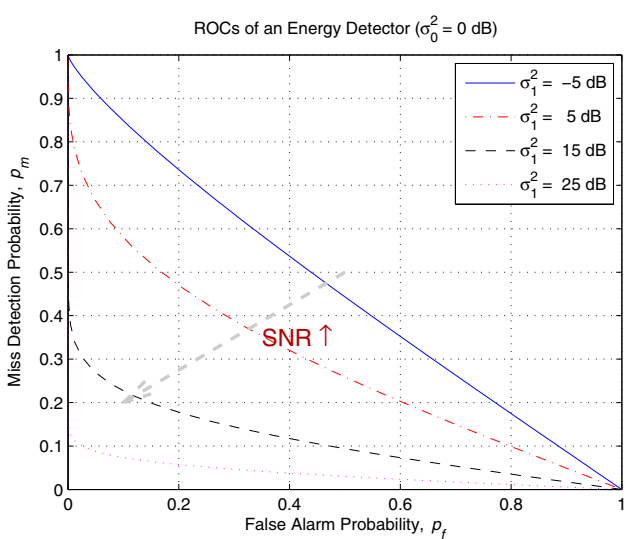

Fig. 4. Receiver Operating Characteristics of an Energy Detector in a CSMA/CA node

characteristic ( $\frac{\partial S}{\partial p_{f}}$ and $\left.\frac{\partial S}{\partial p_{m}}\right)$. This separation makes it easy to consider other types of carrier sensor since a different carrier sensor changes only the detector characteristics. For the energy detector, the detector characteristics $\frac{d p_{f}}{d \eta}$ are $\frac{d p_{m}}{d \eta}$ are obtained from Eqn. (16), and given by

$$
\begin{aligned}
\frac{d p_{f}}{d \eta} & =-e^{-\frac{\eta}{2 \sigma_{0}^{2}}} \cdot \sqrt{\frac{1}{2 \pi \eta \sigma_{0}^{2}}}, \\
\frac{d p_{m}}{d \eta} & =e^{-\frac{\eta}{2\left(\sigma_{0}^{2}+\sigma_{1}^{2}\right)}} \cdot \sqrt{\frac{1}{2 \pi \eta\left(\sigma_{0}^{2}+\sigma_{1}^{2}\right)}} .
\end{aligned}
$$

The detector-independent throughput characteristics $\frac{\partial S}{\partial p_{f}}$ and $\frac{\partial S}{\partial p_{m}}$ can be numerically obtained by differentiating $S$ with respect to $p_{f}$ and $p_{m}$, respectively, using the obtained throughput $S$ in (13) as a function of $p_{f}$ and $p_{m}$. Substituting Eqns. (18) and (19) into Eqn. (17), we have

$$
\begin{aligned}
\left|\frac{d}{d \eta} S\right|= & \mid-e^{-\frac{\eta}{2 \sigma_{0}^{2}}} \cdot \sqrt{\frac{1}{2 \pi \eta \sigma_{0}^{2}} \cdot\left(\frac{\partial S}{\partial p_{f}}\right)_{p_{f}=\epsilon(\eta), p_{m}=\delta(\eta)}+} \\
& e^{-\frac{\eta}{2\left(\sigma_{0}^{2}+\sigma_{1}^{2}\right)}} \cdot \sqrt{\frac{1}{2 \pi \eta\left(\sigma_{0}^{2}+\sigma_{1}^{2}\right)}} \cdot\left(\frac{\partial S}{\partial p_{m}}\right)_{p_{f}=\epsilon(\eta), p_{m}=\delta(\eta)} \mid
\end{aligned}
$$

Similar analysis can be done for the matched filter case only by changing the detector characteristics.

\section{Numerical Results}

In this section, we investigate the throughput and the delay as functions of the physical-layer sensing parameters $p_{f}$ and $p_{m}$ as well as other CSMA/CA parameters based on both analysis and simulation. The CSMA/CA parameter setting in our analysis and simulation is shown in Table I. The analytic results are obtained based on the expressions in Section III. Simulation is performed based on the CSMA/CA protocol explained in Section II.

Figs. 5 and 6, respectively, show the normalized throughput $S$ and the mean access delay $D$ for the value of $p_{f}$ from 0 to 1 when $p_{m}$ is fixed to be 0.1 . While the contention window size $W$ is fixed to 64 , the number of users and the frame length $L$ are varied. The solid lines represent the analysis based 
TABLE I

CSMA/CA RELATEd Parameter Values

\begin{tabular}{|c|c|c|}
\hline Parameter & Value & Description \\
\hline \hline$L$ & {$[1,9]$ slots } & Length of Frame \\
\hline$W$ & {$[4,64]$} & Contention Window Size \\
\hline
\end{tabular}

on our model whereas the simulation results are shown with marks. The simulation well matches our analysis. It is seen that the throughput decreases and the delay increases as the false alarm probability increases. This is because CSMA/CA stations become more conservative in accessing the channel. In other words, transmitters lose the chance of successful transmission due to the high sensing threshold. It is also seen that the normalized throughput increases as the the frame length $L$ becomes large. This is because since a longer frame occupies more slots when the transmission is successful in this parameter setting. Note that the $S$ for $n=5$ is higher than that for $n=2$. This implies that the channel is not efficiently used yet when $n=2$ in case of $W=64$. (Of course, all this behavior depends on $L, W$ and $n$.)

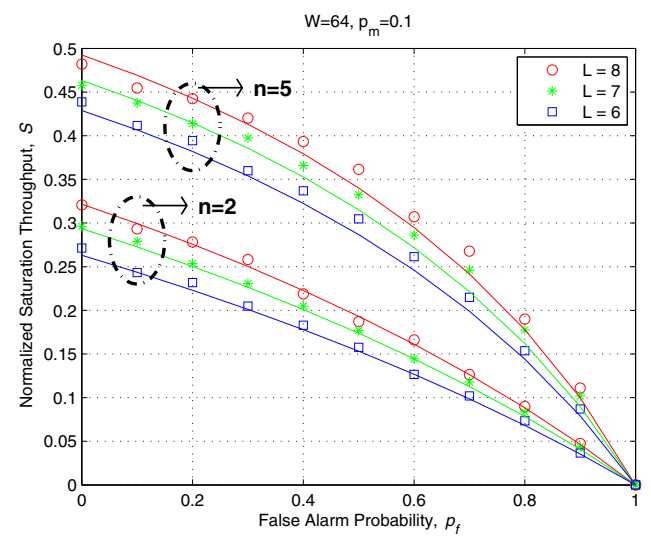

Fig. 5. Normalized Throughput $S$ for varying the false alarm probability with fixed miss detection probability $\left(0 \leq p_{f} \leq 1, p_{m}=0.1\right)$ - solid line: analysis, mark: simulation

Fig. 7 and 8, respectively, show the normalized throughput and the mean access delay for the value of $p_{m}$ from 0 to 1 for a fixed $p_{f}=0.1$. While $W$ is fixed to 32 , the number of users and the frame length are varied. It is seen that the simulation matches our analysis well.

Fig. 9 shows the sensitivity of throughput w.r.t. the sensing threshold, $\frac{d S}{d \eta}$. Here, we varied the frame length $L$ for fixed values of $W$. Fig. 10 shows the sensitivity of throughput, where $W$ is varied for fixed values of $L$. In both cases, the number of users is set to 2 . It is seen that for each value of $W$ there exists a value for $L$ that minimizes the sensitivity w.r.t. the sensing threshold, or vice versa. To investigate the relationship among $L, W$ and sensitivity, we plot the throughput sensitivity w.r.t. the sensing threshold as a function of $L$ and $W$ in 3-D plot, as shown in Figure 11. It is observed that the minimum sensitivity points are well aligned with a linear approximation, i.e., a line $W=\beta^{*} L$ for some $\beta^{*}$. When

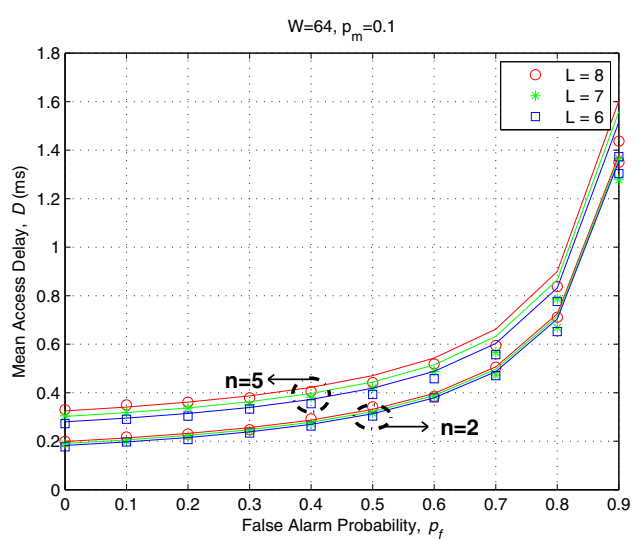

Fig. 6. Mean Access Delay $D$ for varying the false alarm probability with fixed miss detection probability $\left(0 \leq p_{f} \leq 1, p_{m}=0.1\right)$ - solid line: analysis, mark: simulation

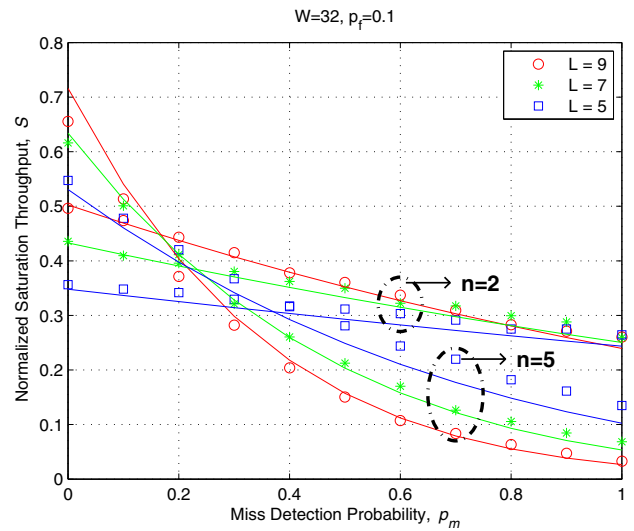

Fig. 7. Normalized Throughput $S$ for varying the miss detection probability with fixed false alarm probability $\left(0 \leq p_{m} \leq 1, p_{f}=0.1\right)$ - solid line: analysis, mark: simulation

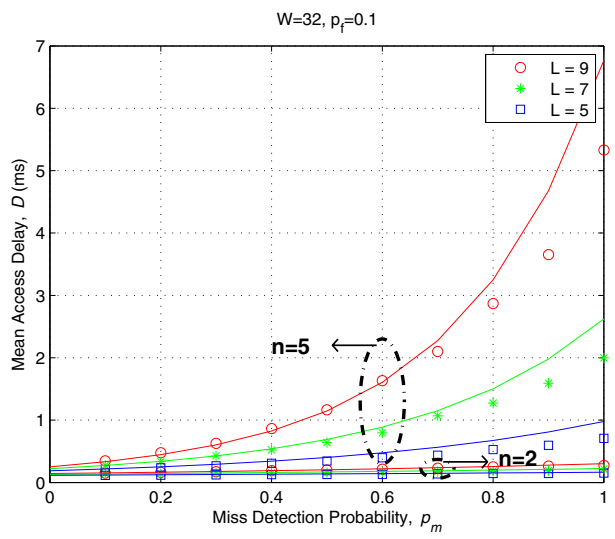

Fig. 8. Mean Access Delay $D$ for varying the miss detection probability with fixed false alarm probability $\left(0 \leq p_{m} \leq 1, p_{f}=0.1\right)$ - solid line: analysis, mark: simulation 
the ratio $W / L$ of the operating CSMA/CA system is away from $W / L=\beta^{*}$, the throughput is very sensitive w.r.t. the sensing threshold. Hence, the ratio $W / L$ can be regarded as the key design parameter for CSMA/CA with sensing error to determine the sensitivity of the system w.r.t. the carrier sensor design at the physical layer. Thus, care should be taken for the carrier sensor design for extreme values of $W / L$ not to degrade the system throughput by poor design of the sensing threshold.

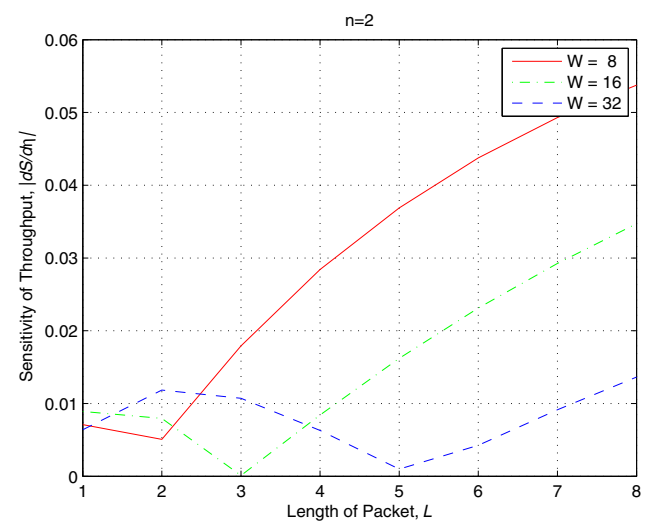

Fig. 9. $\quad \frac{d S}{d \eta}$ for varying $L$ with some sets of fixed $W$ values when $p_{f}$ and $p_{m}$ are set to 0.113 and $0.219\left(\sigma_{0}^{2}=0 \mathrm{~dB}\right.$ and $\left.\sigma_{1}^{2}=15 \mathrm{~dB}\right)$

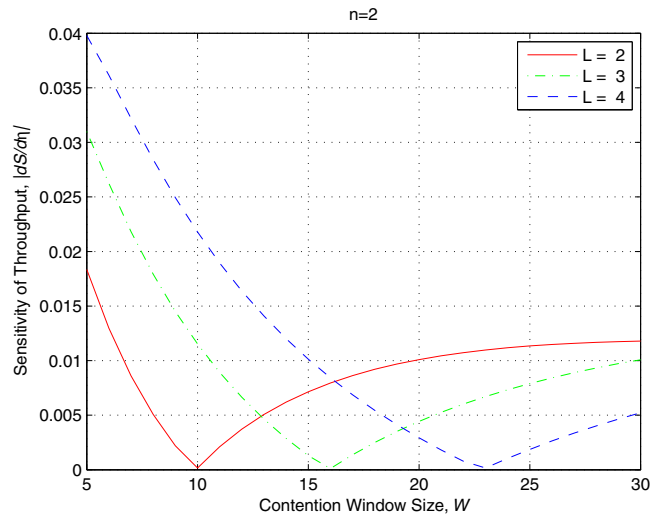

Fig. 10. $\frac{d S}{d \eta}$ for varying $W$ with some sets of fixed $L$ values when $p_{f}$ and $p_{m}$ are set to 0.113 and $0.219\left(\sigma_{0}^{2}=0 \mathrm{~dB}\right.$ and $\left.\sigma_{1}^{2}=15 \mathrm{~dB}\right)$

\section{CONCLUSION}

We have analyzed the performance of the CSMA/CA protocol under carrier sensing error. We have obtained the normalized throughput and the mean access delay using a modified Markov chain model that captures both types of sensing errors: false alarm and miss detection. We have investigated the properties of the throughput and the delay as functions of the false alarm and miss detection probabilities. The behavior of the throughput as a function of the false alarm and miss detection probabilities depends on other CSMA/CA parameters such as the contention window size $W$ and frame

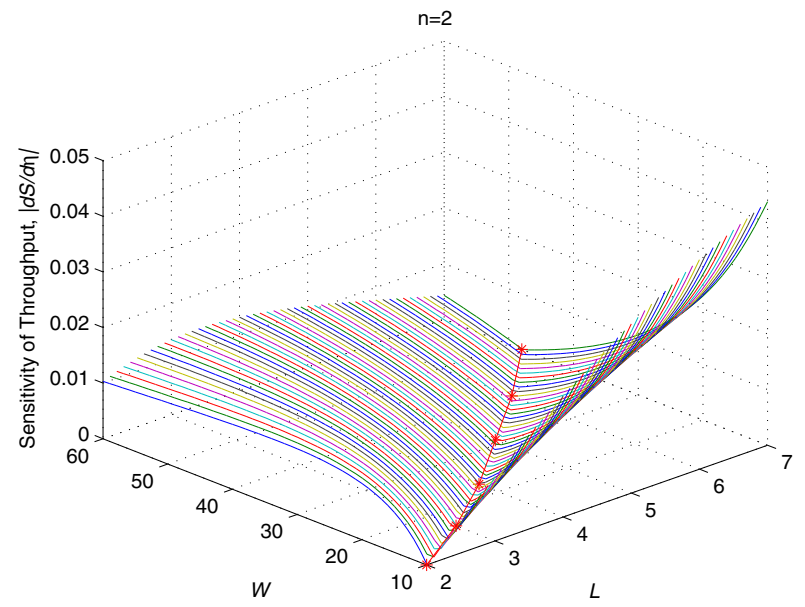

Fig. 11. $\frac{d D}{d \eta}$ for varying $W$ and $L$ when $p_{f}$ and $p_{m}$ are set to 0.113 and $0.219\left(\sigma_{0}^{2}=0 \mathrm{~dB}\right.$ and $\left.\sigma_{1}^{2}=15 \mathrm{~dB}\right)$

length $L$. Simulation are provided to validate our analysis. The ratio $W / L$ is the key parameter to determine the sensitivity of the throughput w.r.t. the channel sensing threshold.

\section{REFERENCES}

[1] J. W. Chong, Y. Sung, and D. K. Sung, "Cross-layer performance analysis for CSMA/CA systems: Impact of imperfect sensing," in submitted to SPAWC 2008, Jan. 2008.

[2] L. Kleinrock and F. Tobagi, "Packet switching in radio channels: Part I - carrier sense multiple-access modes and their throughput-delay characteristics," IEEE Transactions on Communications, vol. 23, no. 2 , pp. 1400-1416, 1975.

[3] WLAN SIG., WLAN Specification Version 2.0, 2004

[4] IEEE 802.15.4, Part 15.4: Wireless Medium Access Control (MAC) and Physical Layer (PHY) Specifications for Low-Rate Wireless Personal Area Networks (LR-WPANs), 2003.

[5] G. Bianchi, "Performance analysis of the IEEE 802.11 distributed coordination function," IEEE Journal on Selected Areas in Communications, vol. 18 , no. 3, pp. 535-547, 2000.

[6] G. Bianchi and I. Tinnirello, "Remarks on IEEE 802.11 DCF performance analysis," IEEE Communications Letters, vol. 9, no. 8, pp. 765-767, 2005.

[7] T. R. Park, T. H. Kim, J. Y. Choi, S. Choi, and W. H. Kwon, "Throughput and energy consumption analysis of IEEE 802.15.4 slotted CSMA/CA," IEEE Electronics Letters, vol. 14, no. 18, pp. 1017-1019, 2005.

[8] C. Y. Jung, H. Y. Hwang, D. K. Sung, and G. U. Hwang, "Enhanced Markov chains model and throughput analysis of the slotted CSMA/CA of the IEEE 802.15.4 under an unsaturated traffic condition," IEEE Transaction on Vehicular Technology (accepted).

[9] L. Krishnamurthy and et al., "Making Radios More Like Human Ears," Mesh Network Summit, 2004 (http://research.microsoft.com/meshsummit/techprogram.aspx).

[10] H. V. Poor, An Introduction to Signal Detection and Estimation, 2nd Edition, Springer, New York, NY, 1994. 